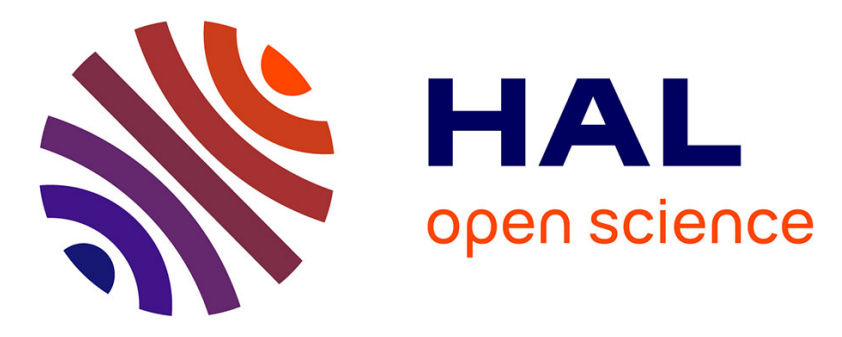

\title{
Transport and Magnetic Measurements on Bi2Sr2CaCu2O8 Nanowire Networks Prepared Via Electrospinning
}

Michael R Koblischka, Xian Lin Zeng, Thomas Karwoth, Thomas Hauet, Uwe Hartmann

\section{- To cite this version:}

Michael R Koblischka, Xian Lin Zeng, Thomas Karwoth, Thomas Hauet, Uwe Hartmann. Transport and Magnetic Measurements on Bi2Sr2CaCu2O8 Nanowire Networks Prepared Via Electrospinning. IEEE Transactions on Applied Superconductivity, 2016, 26 (3), pp.1800605. 10.1109/TASC.2016.2542139 . hal-01569150

\author{
HAL Id: hal-01569150 \\ https://hal.science/hal-01569150
}

Submitted on 26 Jul 2017

HAL is a multi-disciplinary open access archive for the deposit and dissemination of scientific research documents, whether they are published or not. The documents may come from teaching and research institutions in France or abroad, or from public or private research centers.
L'archive ouverte pluridisciplinaire HAL, est destinée au dépôt et à la diffusion de documents scientifiques de niveau recherche, publiés ou non, émanant des établissements d'enseignement et de recherche français ou étrangers, des laboratoires publics ou privés. 


\title{
Transport and Magnetic Measurements on $\mathrm{Bi}_{2} \mathrm{Sr}_{2} \mathrm{CaCu}_{2} \mathrm{O}_{8}$ Nanowire Networks Prepared Via Electrospinning
}

\author{
Michael R. Koblischka, Xian Lin Zeng, Thomas Karwoth, Thomas Hauet, and Uwe Hartmann
}

\begin{abstract}
Superconducting nanowire networks of $\mathrm{Bi}_{2} \mathrm{Sr}_{2} \mathrm{CaCu}_{2} \mathrm{O}_{8}$ (Bi-2212) were fabricated by means of the electrospinning technique. The electrospinning technique enables the growth of long nanowires up to the millimeter range, whereas the diameter of the nanowires can be controlled by the processing parameters. The resulting materials are fabric-like structures of about $4 \times 4 \mathrm{~mm}^{2}$ in size, showing a large number of interconnects and junctions between the nanowires. The resulting nanowires are of granular nature with a grain size similar to the wire thickness of about $100-150 \mathrm{~nm}$, and the diameter of the nanowires is about 100-200 $\mathrm{nm}$ as determined by electron microscopy. As these nanowire networks are a new class of superconducting materials, we studied the electric transport properties (resistance, $U / I$ characteristics) of such nanowire networks in applied magnetic fields $(0-12 \mathrm{~T})$. Measurements of the susceptibility and magnetization hysteresis loops were performed by SQUID magnetometry as well. Possible applications of such nanowire networks are discussed.
\end{abstract}

Index Terms-Bi-2212, magnetization, magnetoresistance, superconducting nanowire networks.

\section{INTRODUCTION}

$\mathbf{N}$ EW kinds of high-temperature superconductors (HTS) may be promising materials for novel types of applications of superconductivity. In the present work, we present such a new class of HTS superconductors, the fabric-like, non-woven nanowire network. By means of electrospinning [1]-[5], it is possible to prepare HTS superconductor nanowires with considerable lengths of up to the millimeter range, while the wire diameter is about $100-150 \mathrm{~nm}$. These nanowire networks contain numerous interconnects between the individual nanowires, which enable a current flow through the entire sample perimeter in contrast to polycrystalline samples. As superconductor, we have chosen $\mathrm{Bi}_{2} \mathrm{Sr}_{2} \mathrm{CaCu}_{2} \mathrm{O}_{8}$ (Bi-2212) due to the high superconducting transition temperature and the advantageous growth

Manuscript received September 7, 2015; accepted March 4, 2016. Date of publication April 5, 2016; date of current version April 25, 2016. The collabonation Saarbrüclen-Nancy is supported by an EU-INTERREG IVa projoct "GRMN."

M. Koblischka, X. L. Zeng, T. Karwoth, and U. Hartmann are with the Institule of Experimental Physics, Sarland University, 66041 Sararbïcken, Germany (c-mail: m.koblischka@ 1 mx.uni-saurland.de).

T. Hauct is with the Institut Jean Lamour, Universite de Lorraine (UMR CNRS), 54506 Vandavie-lès-Nancy, France.

Color versions of one or more of the figures in this paper are available online at htipd/fiecoxplore.iece.ong.

Digital Object Identifer 10.1109/TASC 2016.2542139 properties, where the crystals show a needle-like structure enabling the fabrication of superconducting wires with high critical current densities [6]. Bi-2212 nanowires were grown recently by Duarte et al. using the same technique [7], but in the current work we focus on the magnetic and electric properties of the nanowire networks.

\section{Experimental Means}

Bi-2212 nanowire networks were grown by the electrospinning technique, employing acetate powders of all constituents and PVP dissolved in deionized water. A heat treatment is required to remove all organic material from the as-prepared nanowire networks and to form the superconducting phase. Finally, an oxygenation step in pure $\mathrm{O}_{2}$ is required. X-ray analysis confirmed that the samples are pure $\mathrm{Bi}-2212$ phase with some residing carbon. Details of the nanowire fabrication procedure are given elsewhere [8], [9]. The constituent phases of the samples were determined by means of a highresolution automated RINT2200 X-ray powder diffractometer, using $\mathrm{Cu}-\mathrm{K}_{\alpha}$ radiation generated at $40 \mathrm{kV}$ and $40 \mathrm{~mA}$. SEM imaging was performed using a Hitachi $\mathrm{S} 800$ scanning electron microscope at $10 \mathrm{kV}$, and the TEM analysis was performed by JEOL JSM-7000 F transmission electron microscope at $200 \mathrm{kV}$ with a $\mathrm{LaB}_{6}$ cathode. Fig. 1 presents an optical image of a nanowire network (a) and an SEM image (b). Furthermore, a view of the entire nanowire fabric is given in the inset to (a). The superconducting properties of the nanowires were confirmed using SQUID magnetometry (Quantum Design MPMS7-XL) and transport measurements were performed using an Oxford Instruments Teslatron 10/12 T cryostat system. A quasi fourpoint measurement was established (see the inset to Fig. 7), and the electric contacts to the samples sputter-coated with an $\mathrm{Au}$ layer were fabricated using copper wires and silver paint.

\section{Results and Discussion}

Firstly, we focus on the magnetization data of the nanowire networks. Fig. 2 presents the superconducting transition (FC/ZFC) measured in an applied field of $1 \mathrm{mT}$. The onset of superconductivity takes place at $75 \mathrm{~K}$, which is somewhat lower than the bulk $T_{c}$ of the Bi-2212 phase ( $85 \mathrm{~K}$ ), which was also observed in Ref. [7]. Clearly visible is further the onset of irreversibility at $\sim 70 \mathrm{~K}$. 

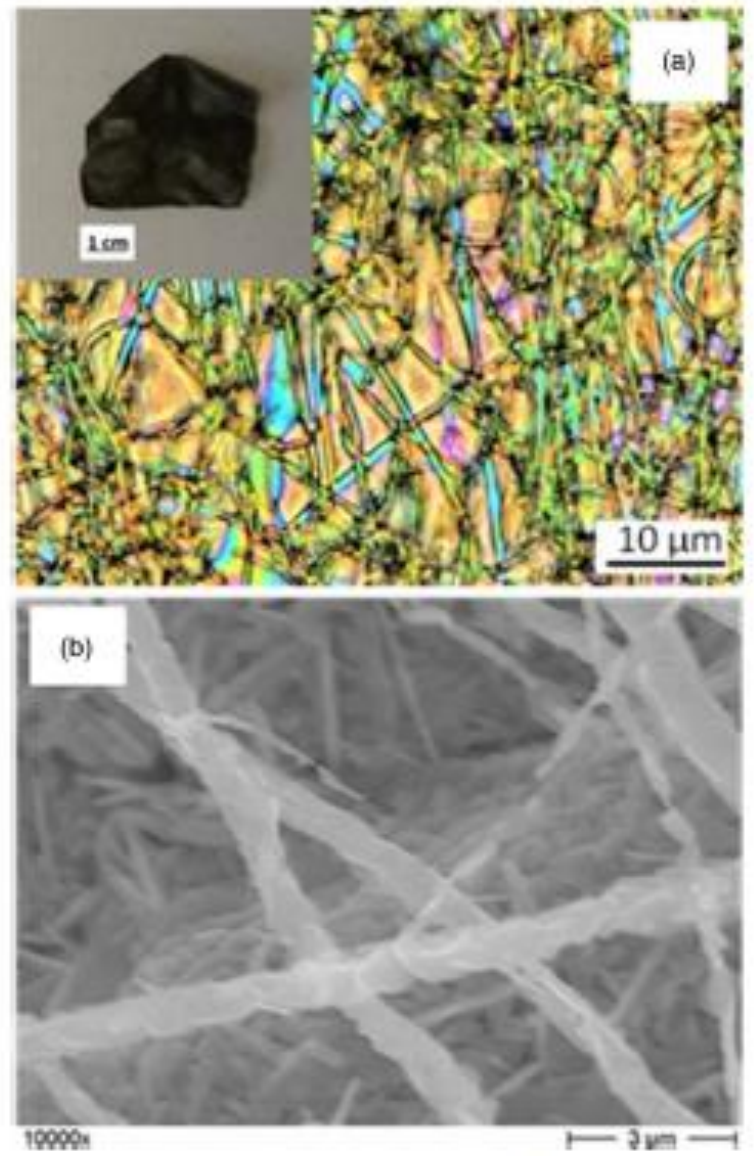

Fig. 1. (a) Optical image of a nanowire network. The inset shows the cntire sample. (b) SEM image of the nanowires, revealing the polycrystalline chaneter of the nanowires and details of the elongated $\mathrm{Bi}-2212$ grains.

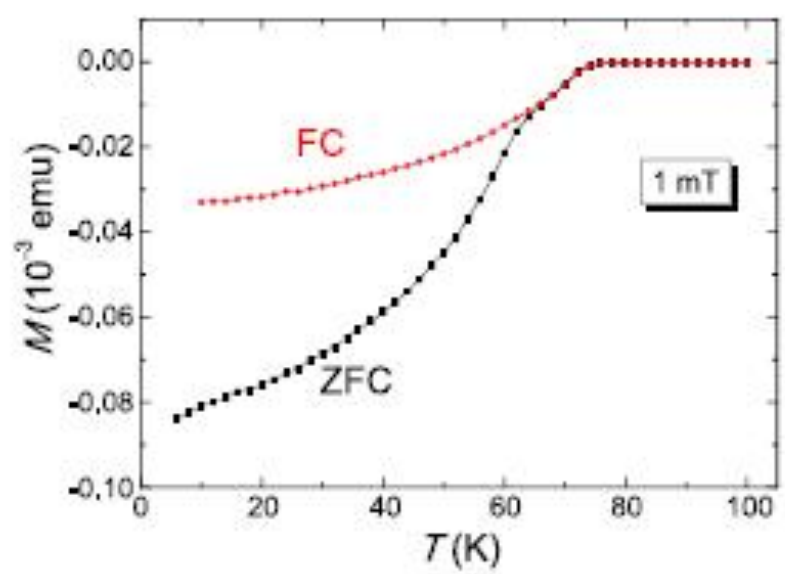

Fig. 2. $M(T)$-diagram measured in ficld cooling (FC) and zero-field cooling ( $/$ FC) in an applied field of $1 \mathrm{mT}$. The enset of superconduxtivity takes place at $\sim 75 \mathrm{~K}$.

Fig. 3 present the magnetization loops measured in fields up to $\pm 7 \mathrm{~T}$ at temperatures ranging from $5 \mathrm{~K}$ up to $70 \mathrm{~K}$. The loops clearly reveal the polycrystalline character of the nanowires. At temperatures above $15 \mathrm{~K}$, the loops get fully asymmetric, and as a consequence, it is not possible to apply the common Bean formalism [10] to obtain the critical current density from the

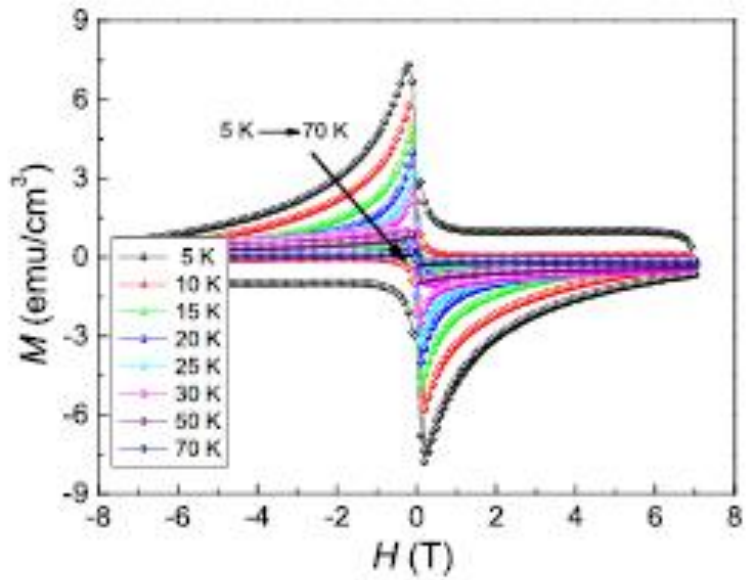

Fig. 3. Magnetization $M$ as function of the applied ficld for temperatures ranging from $5 \mathrm{~K}$ is $70 \mathrm{~K}$.

$M(H)$ data. In order to solve this problem, a modeling of the entire $M(H)$-loop is required. Using the approach of Senoussi of a three-current model [11], where the entire magnetization is composed of three contributions of the grains, the Josephson contacts between them and the shielding of the entire sample, it is possible to model the shape of the measured loops. In fact, our nanowire networks are even more complicated due to the numerous interconnects between the individual nanowires. This contribution is modeled by the respective parameters.

The method to calculate $J_{m}$ by fitting the whole $M(H)$ curves is based on the assumption that the critical current density behaves exponentially on the external field, and the magnetic moment is described by the critical current $J_{m}$, the external field $H$, an effective radius $R$, and some characteristic parameters $H_{0}, R_{0}$, which are determined by the magnetic behavior of the sample. In the field sweep process, the grains of the sample start to decouple when the external field is higher than $H_{0}$. The characteristic length $R_{0}$ is always of the order of the Josephson penetration depth. The $M(H)$ curve can be then fitted in the first, third quadrant (left) and the second, fourth quadrants (right) can be fitted by the equations below:

$$
\begin{aligned}
& M=\frac{J_{m}(H) R}{30}\left(1-\frac{e^{-h}}{4 r_{0}}+\frac{e^{-2 h}}{10 r_{0}^{2}}\right) \\
& M=\frac{H_{c 1}}{4 \pi} \frac{\ln \left(\frac{H_{c 1}}{H}\right)}{\ln \left(\frac{\lambda}{\xi}\right)}, H_{c 1}(T)=H_{c 1}(0)\left(1-\left(\frac{T}{T_{c}}\right)^{m}\right)
\end{aligned}
$$

where $h=H / H_{0}, r_{0}=R_{0} / R, \lambda$ and $\xi$ are the penetration depth and the coherence length, respectively. The constant $m$ is usually 2.2 in the fitting [11], and the parameters of the fit to the data at $5 \mathrm{~K}$ as shown in Fig. 4 are $H_{0}=3.11 \mathrm{~T}$, $H_{\mathrm{c} 1}(0)=16.9 \mathrm{~T}, R=940 \mu \mathrm{m}$, and $R_{0}=233 \mu \mathrm{m}$.

Another important parameter of a superconductor is the irreversibility field, $H_{\text {irr }}$. From the $M(H)$-loops, $H_{\text {irr }}$ was determined using a criterion of $10^{4} \mathrm{Acm}^{-2}$. This procedure works well with the exception of the highest temperatures. The result is presented in Fig. 5. It can be seen that the irreversibility field decreases monotonously on rising temperature. The data are 


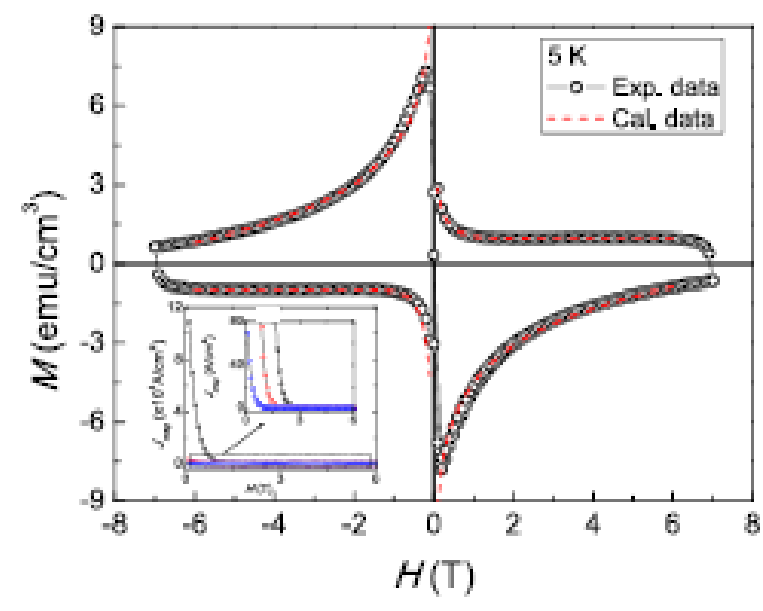

Fig. 4. Magnetization $M$ as function of the applied field at $T=5 \mathrm{~K}$ and the calculated loops using the throe-current model in [11].

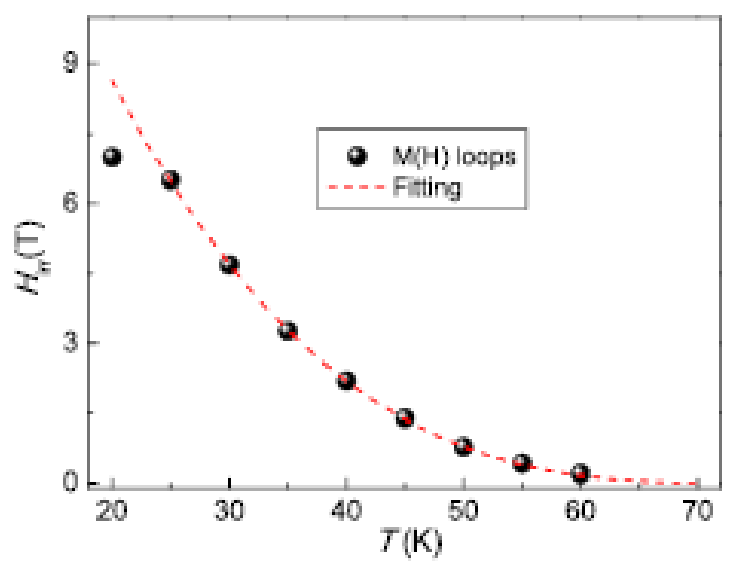

Fig. 5. Irreversibility field $H_{\text {irr }}$ as function of iemperature. The experimental data are fitted well by the function $H_{\mathrm{irr}}=A \times\left(1-T / T_{c}\right)^{3}$ with $A=$ $H_{\text {irr }}=22.1 \mathrm{~T}$.

fitted well by the function $H_{\text {irr }}=A \times\left(1-T / T_{c}\right)^{3}$ [12], which was also observed by various authors on $\mathrm{Bi}-2223$ and $\mathrm{Bi}-2212$ samples. By means of the fitting, the parameter $A=H_{\text {irr }}(0)$ is about $22.1 \mathrm{~T}$ and the critical temperature is determined to be $74.4 \mathrm{~K}$, corresponding to the result from the $M(T)$ curve. At temperatures below $20 \mathrm{~K}$, the available magnetic field of $7 \mathrm{~T}$ is not strong enough to determine $H_{\mathrm{irr}}$, and above $60 \mathrm{~K}$, it is difficult to determine the closing of the loops. The reason of this behavior will be investigated further.

Now, we will turn to the resistance measurements performed on the nanowire networks. Fig. 6 presents the resistancetemperature measurement results at different fields ranging from $0 \mathrm{~T}$ to $10 \mathrm{~T}$. The applied current was $50 \mu \mathrm{A}$. A strong dependence of the resistance behavior on field is shown. In general, the sample shows a behavior of the superconducting transition which agrees well with the one of superconductor nanowires. Two steps in the resistance behavior are observable. Compared to the magnetic measurements as presented in Fig. 2, the onset transition temperature of around $76 \mathrm{~K}$ in the zero field $R(T)$ curve is consistent with $T_{c}$ obtained from the zero-field cooling $M(T)$ curve, thus the drop in the resistance is due to the

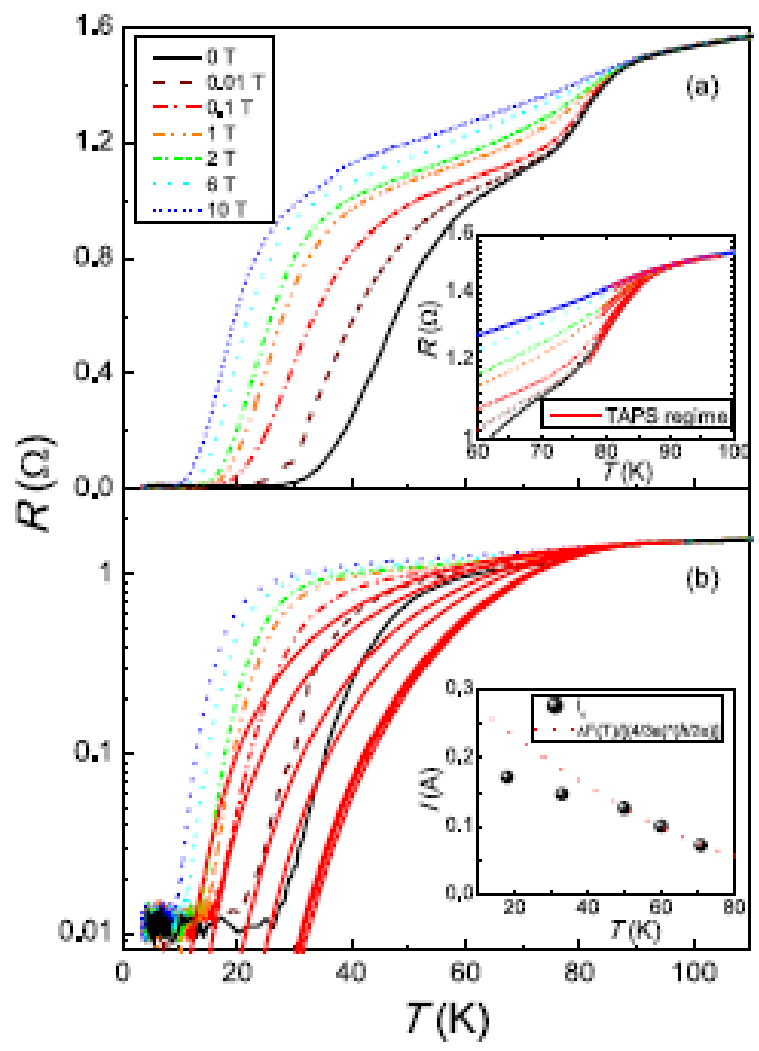

Fig. 6. (a) Resistance $R$ as function of temperature $T$ measured in folds from $0 \mathrm{~T}$ up to $10 \mathrm{~T}$. The inset shows a fit (bold lines) to the data by the TAPS model. (b) $R$ on the logarithmic scale as function of iemperature $T$ from $0 \mathrm{~T}$ up to $10 \mathrm{~T}$, including the fitting lines by the TAPS model. The inset shows the comparison of the measurced $I_{c}(T)$ data and the curve $\Delta F(T) /[(4 / 3 a)(h / 2 e)]$. The curve is found to match the data only above $50 \mathrm{~K}$.

phase transition from the normal state to the superconducting state. In contrast, the non-zero resistance around $T_{c}$ can be attributed to the thermal activated phase slip (TAPS), which can be proven by fitting the data in the respective region using the expression $\exp (-\Delta F / k T)$ [see the inset to Fig. 6(a)]. $\Delta F$ is the energy required to locally suppress superconductivity, following the temperature dependence $\Delta F(T) \approx \Delta F(0) \times(1-$ $\left.T / T_{c}\right)^{3 / 2}[14]$, where $\Delta F(0)=27.2 \mathrm{meV}$ at $0 \mathrm{~T}$. As expected, when the temperature gets lower, the resistance behavior deviates from the TAPS fitting which can be seen in Fig. 6(b), indicating that TAPS could not explain the resistance behavior far away from $T_{c}$. The non-zero resistance at the second step in the resistance (ranging from 40 to $80 \mathrm{~K}$ in the curve at $0 \mathrm{~T}$ ) is due to the normal state resistance $R_{N}$, caused by the intergrain weak links inside the individual nanowires [16]. According to our data, the onset temperature of the first resistance drop decreases faster than the onset temperature of the second drop on the increasing field, which indicates that $T_{c}$ is more sensitive to the field than to the variation of $R_{N}$. At low temperatures near $T=4 \mathrm{~K}$, the resistance is small but non-zero, which can be related to the scattering of charge carriers at the grain boundaries (GBs).

Fig. 7 shows the $U(I)$ measurement of the nanowire network at various temperatures ranging from $18 \mathrm{~K}$ to $100 \mathrm{~K}$. It can be found that below the $T_{c}$ of the sample $(18 \mathrm{~K}$ to $71 \mathrm{~K}$ ), a 


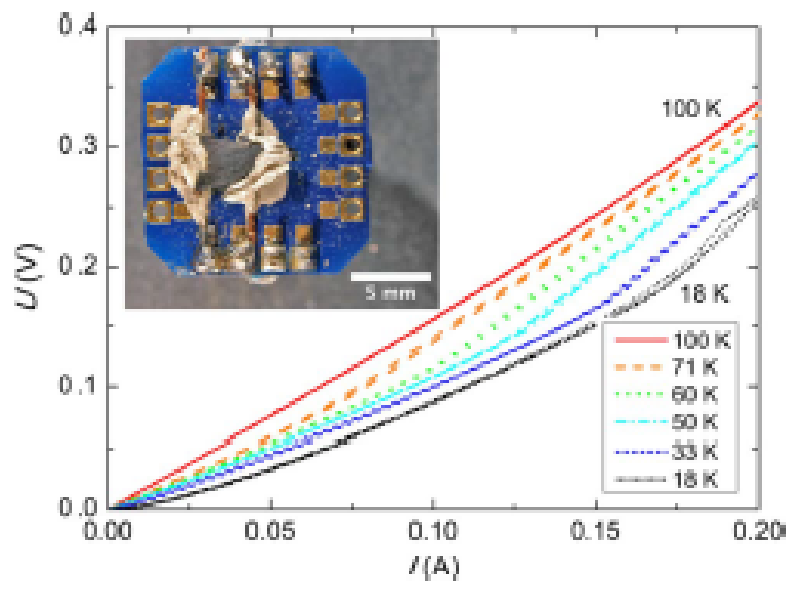

Fig. 7. $U / I$ chanacteristics of the Bi-2212 nanowire network moasured at various temperatures between $18 \mathrm{~K}$ and $100 \mathrm{~K}$. The inset presents the nanowire network with the electric contacts.

transition from the superconducting to the normal state regime occurs, even though the superconducting regime shows a fairly high residual resistance. This may also be attributed to the high amount of GBs inside the nanowire network which correlates to the observations of the $R(T)$ data. The critical current for this transition increases on decreasing the temperature from $0.072 \mathrm{~A}$ to $0.172 \mathrm{~A}(0.072 \mathrm{~A}$ at $71 \mathrm{~K}, 0.101 \mathrm{~A}$ at $60 \mathrm{~K}, 0.126 \mathrm{~A}$ at $50 \mathrm{~K}$, $0.147 \mathrm{~A}$ at $33 \mathrm{~K}, 0.172 \mathrm{~A}$ at $18 \mathrm{~K}$ ). Considering the complicated microstructure of the nanowire network, a determination of the effective radius of our sample is not possible, thus it is not possible to obtain the critical current density directly from the critical current data.

Tinkham has demonstrated the relationship between $I_{c}(T)$ and $\Delta F(T)$ that [15]:

$$
\Delta F(T)=\frac{4}{3 a}\left(\frac{\hbar}{2 e}\right) I_{c}(T) .
$$

From the comparison of the $I_{c}(T)$ data and the result of $\Delta F(T)$ from the TAPS fitting, it can be seen in the inset graph of Fig. 6(b) that $\Delta F(T)$ only matches the $I_{c}(T)$ data above $50 \mathrm{~K}$, which is in agreement with the former conclusion that the TAPS model does not fit the result far from $T_{c}$. In equation (3), $a$ is a constant of order unity. In our case, the value of $a$ is found to be $2.72 \times 10^{4}$, which is much larger than the result of [14]. Their nanowires are fabricated by e beam lithography using a superconducting thin film. The granular effect stems mainly from the fixed grain boundary. So, less inner stress can be induced by an applied field, thus the resistance behavior does not vary much with the field. In contrast, our nanowire network is much more sensitive to applied fields as shown in Fig. 6 due to the numerous GBs and the interconnects between the individual nanowires. Furthermore, in our case the critical currents are nearly 1000 times higher, which may explain the difference in $a$. The influence of the granular weak link structure, and the real mechanism of this behavior will be further investigated. The result of our $\mathrm{Bi}-2212$ nanowire network sample at least proves the statement that cuprate superconductivity shows a weak-coupling BCS behavior.
Besides the importance of such superconducting nanowires for basic research [17]-[20], nanowires of superconducting materials and their networks are also interesting objects for several types of applications. Individual superconducting nanowires may serve as superconducting nanoelectronic components or in devices for photodetection [14], [21]. The possible applications of nanowire network fabrics may be twofold: Firstly, the nanowire networks can be used as building blocks for the production of larger, bulk samples with a specifically designed microstructure as already discussed in [1]. This requires, however, the production of a large enough number of nanowires, which can be fulfilled by employing the electrospinning technique. A respective preparation route is especially interesting for new types of bulk superconducting samples with nanoengineered porosity. Several new types of such samples which were already presented in the literature consist of superconducting fabrics [22] and superconducting foams [23]-[26]. The nanoporosity of the bulk superconductors offers various advantages like an easier oxygenation process, weight reduction, and easier cooling [23], [26]. In this sense, one may apply the present nanowire networks as building blocks for future bulk superconductors with controlled nanoporosity. A second type of application of electrospun nanowire networks may take place wherever the weight of the superconducting material is counting. As discussed already in [22] presenting the superconducting fabrics, such materials may see use as active magnetic shielding or in places where it is required to cover a complicated sample geometry. Therefore, the fabrication and testing of the non-woven superconducting nanowire networks is an important issue to understand the properties of this new class of superconducting material.

\section{Conclusion}

Non-woven nanowire networks of the high-temperature superconductor $\mathrm{Bi}-2212$ have been fabricated using the electrospinning technique. These nanowire networks are characterized by the long length of the nanowires, causing numerous interconnects between the individual nanowires and thus enabling the flow of supercurrents through the entire sample perimeter. The samples were characterized by means of SQUID magnetometry and transport measurements, revealing details of their arrangement within the fabrics. Due to the numerous interconnects, the materials show granular hysteresis loops, which can only be evaluated applying a modeling of the entire loop. The temperature behavior of the irreversibility field is found to follow the same law as bulk Bi-2212 samples. The resistance and $U / I$-characteristics measured in applied magnetic fields up to $10 \mathrm{~T}$ demonstrate the behavior of the intergrain and inter-wire weak links.

\section{ACKNOWLeDgMENT}

We thank V. Presser (INM Saarbrücken and Saarland University) for the possibility to use the electrospinning apparatus, and J. Schmauch (Saarland University, group Prof. Birringer) for performing the TEM analysis. 


\section{REFERENCES}

[1] Y. Huang, X. F. Duan, Q. Q. Wei, and C. M. Lieber, "Directed assembly of one-dimensional nanostructures into functional networks," Science, vol. 291, no. 5504, pp. 630-633, Jan. 2001.

[2] H. Wu, W. Pan, D. Lin, and H. Li, "Flectrospinning of ceramic nanofibers: Fabrication, assembly and applications," I. Adv. Ceram., vol. 1, pp. 2-23, 2012.

[3] M. Law, J. Goldberger, and P. D. Yang, "Semiconductor nanowires and nanotubes," Ann. Rev. Mater. Res., vol. 34, pp. 83-122, 2004.

[4] D. L.i, T. Herricks, and Y. N. Xiz, "Magnetic nanofibers of nickel ferriteprepared by clectrospinning." Appl. Phys. Lett., vol. 83, pp. 4586-4588, 2003.

[S] D. Li, J.T. MeCann, and Y. N. Xia, "Electrospinning: A simple and versatile technique for producing ceramic nanofibers and nanotubes," $J$. Amer Ceram. Soc., vol. 89, pp. 1861-1869, 2006.

[6] F. Kametani et al, "Comparison of growth texture in round $\mathrm{Bi}_{2} \mathrm{Sr}_{2} \mathrm{CaCu}_{2} \mathrm{O}_{3}$ and flat $\mathrm{Bi}_{2} \mathrm{Sr}_{2} \mathrm{Ca}_{2} \mathrm{Cu}_{3} \mathrm{O}_{10}$ wires at its relation to high critical current density development," Sci. Rep., vol. 5, 2015, Art. no. 8285.

[7] E. A. Duarte, P. A. Quintero, M. W. Meisel, and J. C. Nino, "Electrospinning synthesis of superconducting BSCCO nanowires," Phys. C. vol. 496 pp. 109-113, 2013.

[8] J. M. Li, X. L Zeng, A. D. Mo, and Z. A. Xu, "Fibrication of cuprate supereonducting $\mathrm{L} .21 .85 \mathrm{Sm} .15 \mathrm{CuO}_{4}$ nanofibers by electrospinning and subsequent calcination in oxygen," CrystEngComm, vol. 13, pp. 6964-6967, 2011

[9] X. L. Zeng, M. R. Koblischkn, and U. Hartmann, "Synthesis and characterization of electrospun superconducting $(\mathrm{L} \cdot 2, \mathrm{Sr}) \mathrm{CuO}_{4}$ nancrwires and nanoribbons," Mater. Res. Exp., vol. 2, 2015, Art. no. 095022.

[10] C. P. Bean, "Magnetization of hard superconductors," Phys. Rev Left. vol. 8, pp. 250-253, 1962.

[11] S. Senoussi, "Review of the critical current densities and magnetic irreversibilities in high- $T_{c}$ superconductors," $J$. Phys. III France, vol. 2, pp. 1041-1257, 1992.

[12] M. R. Koblischka and J. Sosncrwski, "Temperature-dependent scaling of pinning force data in Bi-based high-T superconductors," Eur Phys. J. B, vol. 44, no. 3, pp. 277-280, Apr. 2005.

[13] M. Tinkham, C. N. Ian, and N. Markovic, "Resistance induced by quantum phase-slips in superconducting nanowires," Physica E, vol. 18, no. 1-3, pp. 308-311, May 2003.
[14] K. Xu and J. R. Heath, "L.ong, highly-ondered high-temperature superconductor nanowire arrays," Nano Lett., vol. 8, no. 11, pp. 3845-3849, Nov. 2008 .

[15] M. Tinkham and C. N. Lau, "Quantum limit to phase coherence in thin superconducting wires," Appl. Phys. Lett, vol. 80, no. 16, pp. 2946-2948, 2002.

[16] A. T. Bollinger, A. Rogachev, M. Remeika, and A. Berryadin, Fiffect of morphology on the superconductor-insulator transition in 1-dimensional nancrwires," Phys. Rev. B, Condens. Matter, vol. 69, 2004, Art. no. 180503 .

[17] A. Bezryadin, C. N. L.au, and M. Tinkham, "Quantum suppression of superconductivity in ultrathin nanowircs," Nature, vol. 404, pp. 971-974, 2002.

[18] D. Y. Vodolazov, F. M. Pecters, L. Piraux, S. Matefi-Tempfli, and S. Michotte, "Current-voltage characteristics of quasi one-dimensional supereonductors: An S-shaped curve in the constant voltage regime," Phs. Rev. Lett., vol. 91, 2003, Art. no. 157001.

[19] M. Zgirski, K.-P. Riikonen, V. Touboltsev, and K. Arutymov, "Size dependent breakdown of supercondectivity in ultranarrow nanowires," Nano. Lett. vol. 5, pp. 1029-1033, 2005.

[20] J. Wang et af., "Magnetoresistance oscillations of ultuthin Pb bridges," Nano Res., vol. 2, pp. 671-677, 2009.

[21] R. Arpaia, S. Navay, F. Lombardi, and T. Bauch, "Improved nanopatterning for YBCO nancrwires approaching the depairing current," IEEE Trans. Appl. Supercond, vol. 23, no. 3, Jun. 2013, Art. no. 1101505.

[22] E. S. Reddy, J. G. Noudem, M. Tarka, and G. J. Schmitz, "Monodomain YBCO superconductor fabrics prepared by an infiltration process," Suppercond. Sci. Technol., vol. 13, no. 6, pp. 716-721, 2000.

[23] E. S. Reddy and G. J. Schmitz, "Superconducting foams," Supencond Sci Technol., vol. 15, pp. 1.21-1.24, 20002.

[24] E. S. Reddy and G. J. Schmitz, "Ceramic foams,"Amer. Ceram Soc. Bull., vol. 81, pp. 35-37, 2002.

[25] P. Ficrtck, B. Andracjewski, and W. Sadowski, "Synthesis and transport properties of porous superconducting ceramics of YBa2 $\mathrm{Cu}_{3} \mathrm{O}_{7}, " \mathrm{Rev}$. Adv Mater Sci., vol. 23, pp. S2-S6, 2010.

[26] E. S. Reddy, N. Hari Babu, Y. Shi, D. Cardwell, and G. J. Schmits, Thocessing of lange grain Y-123 superconductors with pre-defined porous structures," Suppercond. Sci. Technol, vol. 18, pp. S1S-S18, 2005. 